\title{
Political ecology de l'engrillagement forestier privé en Sologne : quels sont les enjeux socioenvironnementaux au cœur du conflit ?
}

\author{
Marie Baltzinger ${ }^{1}$, Juliette Mouche ${ }^{2}$, Marieke Blondet ${ }^{3}$, Baptiste Hautdidier ${ }^{4}$ \\ 1 Écologie forestière, IRSTEA, UR EFNO, 45290 Nogent-sur-Vernisson, France \\ 2 Anthropologie de l'environnement, AgroParisTech, 75231 Paris Cedex 05, France \\ 3 Anthropologie sociale, MNHN, UMR7206 Éco-anthropologie et ethnobiologie, Paris, France \\ 4 Géographie, IRSTEA, UR ETBX, 33612 Cestas, France
}

\begin{abstract}
En Sologne, des grillages limitent, barrent, orientent, définissent des intérieurs et des extérieurs au sein même de la forêt. Cette politique d'engrillagement fait débat et suscite des controverses, ce qui a conduit le conseil régional de la région Centre à soutenir un projet de recherche. Une équipe de recherche interdisciplinaire (écologues, anthropologues et géographes) a pu analyser les conséquences d'un tel quadrillage sur les comportements des ongulés et la dynamique des populations qui en résulte, mais aussi sur les relations sociales que ces pratiques engendrent et transforment. En ce sens, le processus d'engrillagement est perçu comme un dispositif de mise en relation et en mouvement des êtres humains et non humains, à la fois dans ses effets sur la population de cerfs et comme manifestation de certaines relations de pouvoir.
\end{abstract}

La Rédaction

Mots-clés :

biodiversité ; cerf ; clôtures ;

environnement ; sanglier

\section{Keywords:}

biodiversity; deer; fences; environment; wild boar

\begin{abstract}
Résumé - Cet article propose une approche interdisciplinaire visant à étudier la dynamique d'engrillagement en Sologne sous ses aspects humains et écologiques. Des travaux ont été menés, relatés par ailleurs dans des manuscrits soumis à des revues d'écologie, afin de décrire le comportement spatial des cerfs de Sologne, en présence de grillages. Un travail d'enquête a également été effectué, portant sur les valeurs associées à l'environnement en Sologne telles qu'elles étaient mises à jour par l'évocation des engrillagements. Nous utilisons ici une approche de type political ecology pour mettre en regard ces deux types de résultats. Nous mettons en évidence deux discours concurrents sur les formes de légitimation des propriétés cynégétiques, dont l'opposition s'articule autour du remplacement du petit gibier par le grand au cours de ces trente dernières années. Nous expliquons le rôle joué par les engrillagements dans cette mise en concurrence.
\end{abstract}

\begin{abstract}
Political ecology of private forest fencing in the French Sologne: what are the social and environmental issues at stake in the dispute? We propose an interdisciplinary approach designed to study the spread of private fencing in the French Sologne as a socio-ecological phenomenon. We conducted two parallel approaches: a study of how fencing may affect spatial use by deer and an interview campaign in order to elucidate which human values were at stake in the fencing debate. To this end we applied a Political ecology approach in order to articulate these two kinds of results. We highlighted two competing discourses regarding hunting-oriented private properties, showing that this contention is based on the replacement of game birds by big game over the past thirty years, which went along with the spread of fencing.
\end{abstract}

La Sologne couvre 500000 ha entre la vallée de la Loire et la vallée du Cher. Elle se distingue par un couvert forestier étendu, d'importantes populations de gibier et un statut foncier majoritairement privé. Depuis plusieurs dizaines d'années, on assisterait - à en croire la presse locale - à un inquiétant phénomène

Auteur correspondant : M. Baltzinger, marie.baltzinger@irstea.fr 


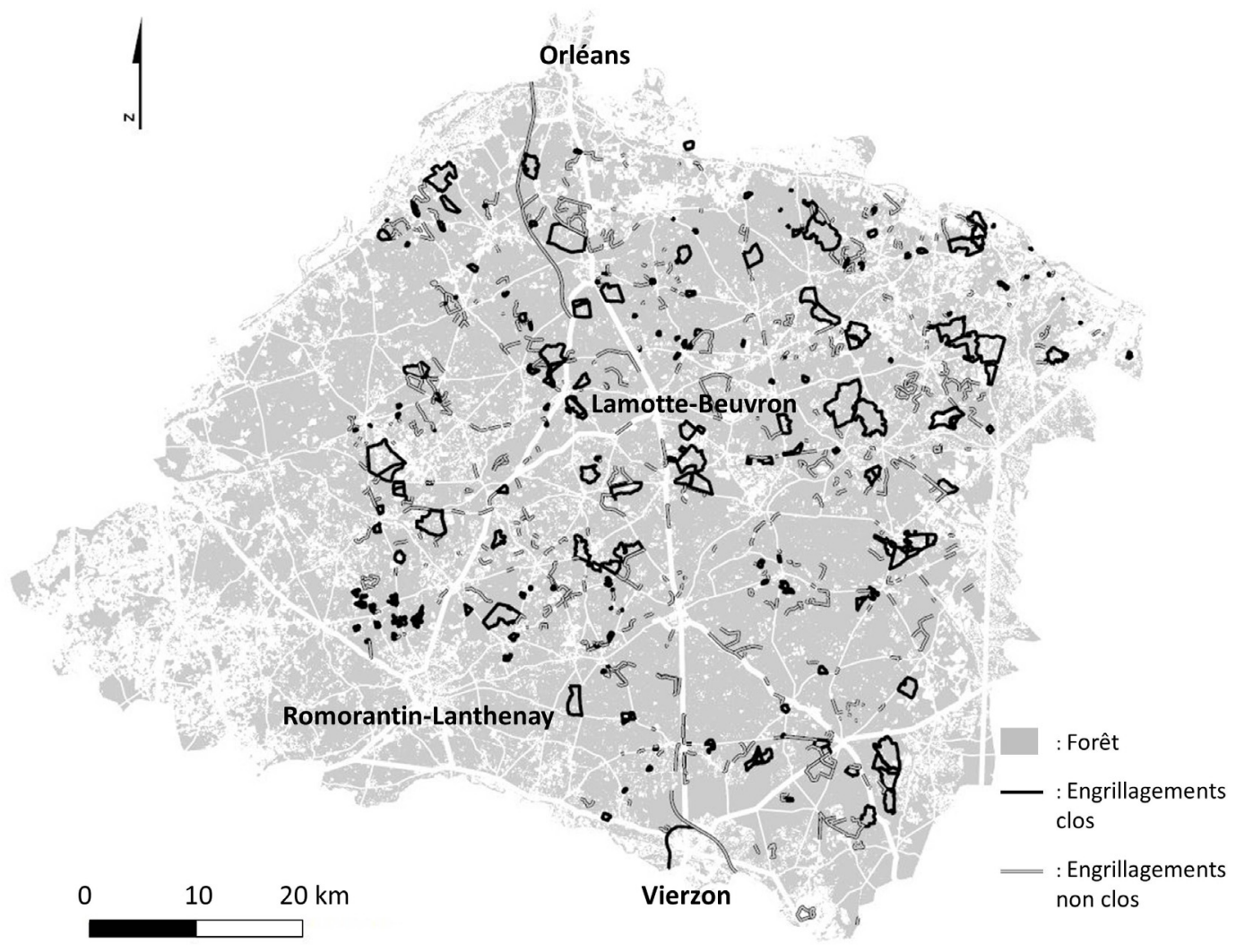

Fig. Carte des engrillagements de Sologne (Sources : couche forestière : IGN, http://inventaire-forestier.ign.fr ; engrillagements : Froissart, 2011).

de morcellement de la Sologne, par la pose de clôtures. Ainsi, en 2012, plusieurs articles dressaient le tableau d'un conflit socioenvironnemental qui se serait cristallisé autour de la question des effets des grillages sur la viabilité et le bien-être des populations de gibier. Cette inquiétude semblait suffisamment brûlante pour que le pays de Grande Sologne ${ }^{1}$ finance une étude intitulée Faire face aux engrillagements en milieu naturel, agricole et forestier en Grande Sologne (Froissart, 2011), laissant penser que les élus de Sologne souhaitaient apporter des solutions au problème généré par les engrillagements. L'étude faisait état d'un inventaire de $670 \mathrm{~km}$ de grillages visibles à partir des routes et des chemins (Fig.). Elle posait également de façon explicite la question de la mobilité du grand gibier en paysage engrillagé, tout en pointant le manque $\mathrm{d}^{\prime}$ informations scientifiques à ce sujet ${ }^{2}$. Alors que les différentes formulations du problème posé par la pré-

\footnotetext{
1 Syndicat mixte réunissant 25 communes, 3 communautés de communes et le conseil général de Loir-et-Cher.

2 Pour cette raison, l'équipe de chercheurs spécialistes de la faune sauvage FONA (Interactions forêt ongulés activités humaines) de l'Irstea à Nogent-sur-Vernisson avait été contactée dès 2010 par Yves Froissart, dans le but d'obtenir des informations permettant d'objectiver scientifiquement cette question.
}

sence de grillages, dans l'étude de 2011 ainsi que dans les médias, évoquaient implicitement l'idée de clôtures hermétiques, la carte produite par Froissart en 2011 (Fig.) dressait une image assez inattendue. En effet, la moitié au moins des grillages répertoriés n'étaient pas hermétiquement clos. Cette importance, à première vue paradoxale, des engrillagements non clos posait la question de leur rôle dans le débat solognot. De ces interrogations multiples est né le projet interdisciplinaire Dynamique spatiale et temporelle de l'engrillagement en Sologne et services écosystémiques (Dysperse ${ }^{3}$ ), qui comprenait deux volets principaux : une étude du comportement spatial des animaux en présence de grillages et une analyse des valeurs associées à l'environnement naturel telles qu'elles se révélaient dans le cadre du débat autour des engrillagements. Ces deux volets complémentaires devaient permettre d'analyser ce débat dans ses dimensions humaines et écologiques. Afin de mettre en regard ces deux types de résultats, nous proposons dans cet article une approche de type political ecology. Nous commencerons par proposer un cadre théorique pour l'étude de la fermeture au moyen de grillages. Nous dresserons

\footnotetext{
3 Le projet Dysperse a été financé par la région Centre de juillet 2012 à décembre 2015, http:/ / dysperse.irstea.fr/.
} 
ensuite un historique des relations entre humains et nonhumains en Sologne avant et après le déploiement des engrillagements. À partir des résultats du volet écologique, nous décrirons le comportement spatial des animaux en espace engrillagé, à plusieurs échelles. Nous expliquerons également comment d'autres pratiques humaines généralement associées aux grillages, notamment la chasse, jouent un rôle dans ces comportements spatiaux. Nous montrerons ensuite comment les engrillagements contribuent à redéfinir les modalités de relations entre non-humains, entre humains et, pour finir, entre humains et non-humains. Nous terminerons en expliquant pourquoi nous considérons les engrillagements de Sologne comme des dispositifs d' « intéressement », $\mathrm{d}^{\prime}$ « enrôlement » et de " mise en mouvement » des acteurs humains et non humains, c'est-à-dire comme des manifestations de pouvoir (Goldman et al., 2011 ; Latour, 2005).

\section{Les engrillagements de Sologne: teichopolitique et teichométrie de la fermeture}

Les concepts dialectiquement liés de « teichopolitique » et « teichométrie » ont été proposés par Ballif et Rosière (2009) afin de décrire la fermeture des territoires, telle qu'elle se manifeste, à toutes les échelles, via la construction de "barrières », terme générique utilisé pour désigner tout type de construction séparatrice. Par teichopolitique, ces auteurs entendent l'ensemble des systèmes visant à contrôler les mouvements, et par teichométrie, la mesure du phénomène d'enfermement non seulement en termes de linéaire mais aussi en termes d'efficacité et d'imperméabilité. En tant que politique, la fermeture implique des acteurs, des idéologies et des objectifs. En tant qu'artefacts matériels, les barrières peuvent être caractérisées par la quantification de l'enfermement qui leur est associé. Le projet interdisciplinaire Dysperse prévoyait, d'une part, de quantifier la fermeture, à plusieurs échelles, notamment en ce qu'elle affectait les acteurs non humains (volet écologique), et, d'autre part, d'analyser la politique de cette fermeture, notamment via l'étude des différentes valeurs qui s'opposaient dans le cadre du débat autour des engrillagements (enquête anthropologique).

D'un point de vue d'écologue, la fermeture renvoie à deux champs différents de la littérature : celui de la conservation (Brechin et al., 2003) et celui de la connectivité (Fahrig, 2003). Alors que le référentiel organisant la science écologique et les politiques de conservation jusque dans les années 1980 reposait plutôt sur la délimitation et le zonage d'espaces de nature remarquables - ce qu'Amelot et al. (2014) vont jusqu'à qualifier de « nature enfermée »-, la connectivité se serait imposée depuis comme un paradigme central en écologie et notamment en biologie de la conservation (Rodary et Bologna, 2014). Définissant la connectivité écologique comme la mesure du degré de relations entre populations d'une même espèce dans des territoires disjoints, Rodary et Bologna considèrent que la problématisation de la connectivité se traduit par des politiques de conservation débordant largement les limites des parcs et réserves. Cette transition d'un paradigme à l'autre est lisible notamment dans le titre d'un ouvrage corédigé par plusieurs spécialistes internationaux de la faune sauvage, paru en 2012 : Fencing for conservation. Restriction of evolutionary potential or a riposte to threatening processes? (Somers et Hayward, 2012). Alors qu'un grand nombre de clôtures, issues du paradigme de la conservation, avaient été conçues pour éviter aux animaux certains dangers engendrés par l'activité humaine (Packer et al., 2013), une littérature écologique plus récente s'interroge sur la fragmentation engendrée par les grillages, notamment lorsque certains habitats d'importance cruciale (points d'eau) deviennent inaccessibles, ou certaines routes de migration impraticables pour les animaux (Holdo et al., 2011). Ces effets des clôtures sont d'autant plus manifestes que l'on s'intéresse à des espèces animales à grand domaine vital comme le cerf élaphe (Cervus elaphus) (Wallis De Vries, 1995). À l'inverse, si les clôtures ne sont pas complètement imperméables, elles peuvent faciliter les déplacements des animaux en les guidant le long d'itinéraires privilégiés (Bergerud et al., 1984). Il a aussi été montré que les engrillagements, même lorsqu'ils ne sont pas bloquants, modifient la répartition des animaux, ce que Webb et al. (2010) interprètent comme une manifestation de «fidélité » des animaux aux espaces partiellement engrillagés. La littérature en écologie laissait donc penser que les effets des engrillagements sur le bien-être, la mobilité et la répartition spatiale des animaux étaient complexes et parfois contradictoires, ce qui justifiait une analyse teichométrique de l'enfermement de ces animaux.

Si l'on s'en tient à la typologie des barrières proposée par Ballif et Rosière (2009), les grillages seraient plus souvent associés à des teichopolitiques de filtrage des flux qu'à des objectifs d'étanchéité forte. De fait, dans le cas de la Sologne, il est rapidement apparu que la question des engrillagements se posait surtout en termes de mobilités : celles des non-humains, mais aussi celles des humains. Intuitivement, nous avions a priori associé les questions de mobilités, notamment celle des cerfs, aux grillages complètement bloquants. Pourtant, la littérature en sciences naturelles, qu'il s'agisse d'éthologie ou d'écologie du paysage, n'éludait pas intégralement la question des barrières filtrantes. Ainsi, s'appuyant sur la notion de site fidelity, Nielsen et al. (1997) et Webb et al. (2010) avaient mis en évidence des modifications de comportements spatiaux chez les cerfs, induites par la 
présence de grillages non clos. Interprétés non plus comme des obstacles mais comme des membranes écologiques, ces dispositifs pouvaient également être assimilés à des membranes exogènes ${ }^{4}$, telles qu'elles avaient été conceptualisées par les écologues du paysage : «Many boundaries originate and are maintained not by exogenous or endogenous forces alone but by an interaction between exogenous and endogenous » (Strayer et al., 2003). Pour ces auteurs, le devenir des membranes écologiques, quelle que soit leur origine, serait lié à leur perméabilité différentielle aux flux d'énergie et de matière circulant au sein de la matrice paysagère et aux interactions biotiques qu'elles permettent ou ne permettent pas. Ces visions d'écologues et d'éthologues semblent, de façon assez baroque, trouver un parallèle dans l'ouvrage d'Olivier Razac, Histoire politique du barbelé (2009). Les murs et les clôtures modernes y sont envisagés comme des dispositifs de maîtrise et de filtrage des flux, produisant et reproduisant une certaine relation à l'espace. Ces infrastructures constitueraient donc des instruments de pouvoir, non parce qu'ils bloquent complètement les flux, mais plutôt parce qu'ils les dirigent et les encadrent. Et l'auteur d'ajouter : "Comprendre notre rapport à l'espace nécessite donc avant tout de comprendre les principes de fonctionnement des dispositifs qui ferment, ouvrent et font communiquer les lieux entre eux. [...] L'espace symbolique, imaginaire et réel produit par ces dispositifs conditionne l'ensemble des exclusions et des hiérarchisations sociales » (Razac, 2009, p. 85). Qu'elle soit issue des sciences naturelles ou des sciences humaines, cette littérature s'accordait donc sur deux aspects : d'une part, les modalités de mobilité induites par l'existence de barrières conditionneraient les mises en relation entre humains et non-humains, d'autre part, les modalités de mise en relation contribueraient en retour à redessiner les barrières, donc les mobilités.

Pour finir, la question autour des engrillagements de Sologne, s'exprimant en termes de mise en relation et de mise en mouvement des êtres humains et non humains, conduisait en filigrane à poser la question des relations de pouvoir. Par relations de pouvoir, nous entendons ici les dispositifs de mise en relation et en mouvement des êtres humains et non humains, au sein de réseaux qui sont eux-mêmes la manifestation de certaines relations de pouvoir entre ces êtres. Pour analyser de tels phénomènes, Callon et Latour (2006 [1981]) proposent de considérer le monde qui nous entoure comme un « collectif » d'acteurs humains et non humains : ces acteurs ne doivent pas être pensés en fonction de leur appartenance à des catégories préexistantes, mais au regard des associations qui les lient les uns aux autres, au sein de ces collectifs ou réseaux. Ces théoriciens considèrent que ce sont les réseaux d'association qui définissent les acteurs,

4 Membranes d'origine humaine. d'où l'utilisation du terme « acteur-réseau » pour désigner l'acteur en ce qu'il est associé à d'autres acteurs au sein de réseaux particuliers. La théorie de l'acteur-réseau désigne par « intéressement », « enrôlement » et «mobilisation » les étapes successives qui permettent à certains acteurs de s'associer à d'autres au sein de réseaux dont ils occupent la position centrale. En effet, c'est la capacité $\mathrm{qu}^{\prime}$ ont certains acteurs à se positionner au centre de réseaux particuliers qui leur permet de manifester et de déployer un surcroît de pouvoir au sein de ces réseaux.

Plus récemment, la théorie de l'acteur-réseau a été mobilisée en political ecology. Il s'agit d'un courant qui s'est imposé au cours des trente dernières années, notamment dans la littérature anglophone, comme une école de pensée interdisciplinaire visant à analyser les relations entre les sociétés et leur environnement dans ce qu'elles présentent de problématique pour les sociétés ou pour l'environnement en question (Benjaminsen et Svarstad, 2009). Les approches de type political ecology n'envisagent généralement pas l'écologie comme une science neutre, qui ne viserait qu'à objectiver des relations universelles entre non-humains, indépendamment des relations entre humains, quelles qu'elles soient. $\mathrm{Au}$ contraire, ces chercheurs pensent que les situations socioenvironnementales problématiques sont considérées comme des problèmes par quelqu'un, pour quelqu'un. Ces approches comprennent les conflits environnementaux comme des discours ${ }^{5}$ concurrents, $c^{\prime}$ est-à-dire comme des tentatives $\mathrm{d}^{\prime}$ " intéressement ", $\mathrm{d}^{\prime}$ "« enrôlement » et de "mobilisation » des mêmes acteurs humains et non humains, dans des réseaux d'architectures différentes (Goldman et al., 2011).

\section{Matériel et méthodes}

Le présent article repose sur une étude interdisciplinaire financée par la région Centre visant à analyser le déploiement des grillages privés sous ses aspects écologiques et humains. Nous avons tout d'abord effectué un travail bibliographique afin de comprendre le contexte historique, géographique et social de la Sologne. Nous avons également cherché à caractériser le contexte médiatique prévalent en 2013, au démarrage de notre projet. Nous avons ainsi analysé le contenu de 18 articles parus dans la presse locale ainsi que celui d'un film, Une maille de trop (Gasselin, 2011), diffusé sur la chaîne de télévision Seasons, afin de mieux comprendre l'origine $\mathrm{du}$ conflit autour des engrillagements. Vingt-trois entretiens semi-directifs (individuels ou collectifs) ont par la suite été menés auprès de 34 acteurs concernés par cette question dans le cadre d'un stage de Master II en anthro-

\footnotetext{
5 Au sens de Latour (2005), c'est-à-dire comme des médiateurs actifs entre humains et non-humains.
} 
Tableau. Caractéristiques des personnes interviewées.

\begin{tabular}{|c|c|c|c|}
\hline Nom & Description de la personne interviewée & Grand propriétaire & Chasseur \\
\hline Saül & Vieille famille d'anciens grands propriétaires & Non & Oui \\
\hline Séraphin & Militant anti-clôtures & Non & Oui \\
\hline Sarah & Militant anti-clôtures & Non & Non \\
\hline Sylvie & Expert forestier & Non & Non \\
\hline Samson & Vieille famille de grands propriétaires & Oui (propriété partiellement engrillagée) & Non \\
\hline Simon & Randonneur & Non & Non \\
\hline Sidonie & Randonneuse & Non & Non \\
\hline Siegfried & Fédération des chasseurs & Oui & Oui \\
\hline Seth & Fédération des chasseurs & Non & Oui \\
\hline Séverine & Fédération des chasseurs & Non & Oui \\
\hline Sophie & Vieille famille de propriétaires & Oui (propriété non engrillagée) & Oui \\
\hline Siméon & Propriétaire première génération & Oui (propriété non engrillagée) & Oui \\
\hline Sulpice & Propriétaire première génération & Oui (propriété partiellement engrillagée) & Oui \\
\hline Sylvestre & Propriétaire première génération & Oui (propriété partiellement engrillagée) & Oui \\
\hline Stéphane & Gérant de propriété & Non & Oui \\
\hline Silviano & Propriétaire première génération & Oui (propriété partiellement engrillagée) & Oui \\
\hline Stanislas & Chasseur & Non & Oui \\
\hline Sven & Gérant de propriété & Non & Oui \\
\hline Sacha & Agent immobilier & Non & Non \\
\hline Samuel & Agent immobilier & Non & Non \\
\hline Salvator & Maire & Non & Non \\
\hline Salomon & Vieille famille de propriétaires & Oui (propriété non engrillagée) & Non \\
\hline Sixtine & Vieille famille de propriétaires & Oui (propriété non engrillagée) & Non \\
\hline Saturnin & Propriétaire première génération & Oui (propriété engrillagée) & Oui \\
\hline Steve & Gérant de propriété partiellement engrillagée & Non & Oui \\
\hline Sullivan & Personnel Office national des forêts & Non & Non \\
\hline Swann & Personnel Office national des forêts & Non & Non \\
\hline Sylas & Personnel Office national des forêts & Non & Oui \\
\hline Servane & Propriétaire première génération & Oui (propriété non engrillagée) & Oui \\
\hline Savin & Propriétaire première génération & Oui (propriété non engrillagée) & Non \\
\hline Serge & Journaliste militant anti-clôtures & Non & Non \\
\hline Sébastien & Expert forestier & Non & Oui \\
\hline Sigisbert & Vieille famille de propriétaires & Oui (propriété non engrillagée) & Non \\
\hline Suzanne & Agricultrice & Non & Non \\
\hline
\end{tabular}

pologie de l'environnement entre avril et juin 2013 (Mouche, 2013) (Tab.). Ce travail d'enquête avait pour but de mettre en lumière les valeurs associées à la nature en Sologne et la façon dont elles étaient affectées positivement ou négativement par l'engrillagement. En parallèle, et dans la mesure où le conflit lié aux engrillagements nous était initialement apparu, à la lecture des médias, comme résultant des effets de ces linéaires sur la grande faune (notamment le cerf), d'autres études - hors de l'objet de cet article - ont été menées afin de comprendre les effets des clôtures sur la population de cerfs en Sologne. À l'échelle du paysage (Sologne), nous avons cherché à caractériser la structuration génétique de cette population et essayé de déterminer si les engrillagements étaient responsables de tout ou partie de cette structuration (Masson, 2015). À l'échelle intermédiaire, nous nous sommes attachés à décrire l'effet des engrillagements sur le choix des domaines vitaux 
par les cerfs. À l'échelle locale (massif forestier), nous avons étudié les effets de différentes densités de cerfs et de sangliers sur la flore du sous-bois et sur l'avifaune (Baltzinger et al., 2016).

\section{La propriété foncière à visée cynégétique dans son contexte historique}

L'étude du cadastre de 1830 a permis à Gillardot d'écrire que, vers 1935, "la plus grande partie de la Sologne était aux mains d'un nombre réduit de grands propriétaires » (Gillardot, 1981, p. 126). Selon cet auteur, les domaines de plus de 100 ha couvraient à l'époque $82 \%$ de la superficie de la région. Cela laisse penser que l'appropriation privée du territoire en Sologne est un phénomène relativement ancien. Par la suite, Édith Chardon (1993) a montré comment, au début du XIX ${ }^{\mathrm{e}}$ siècle, les grands propriétaires de Sologne avaient pu imposer leur objectif cynégétique à la région : pour cette période de référence, elle décrit notamment l'évolution du statut des fermiers, d'agriculteurs locataires à régisseurs ou gardes-chasse salariés. La société solognote était alors très hiérarchisée, organisée autour et en fonction de la chasse au petit gibier, telle qu'elle se pratiquait sur ces grands domaines privés. Elle affirme ainsi que la grande propriété « cont[enait] à elle seule la quasitotalité de la vie économique locale (économie pris au sens large) : agriculture, chasse, pêche, cueillette... jusqu'au braconnage » (Chardon, 1993, p. 210). La légitimité des différents usages sur le territoire était ainsi arbitrée en fonction de leur compatibilité, tant avec la notion de grande propriété privée qu'avec l'objectif cynégétique lui étant associé. Ces différents usages fonctionnaient comme des pratiques socialement structurantes: dans la mesure où l'organisation des journées de chasse, l'élevage du gibier, l'entretien de milieux ouverts et nourriciers, d'étangs et de zones humides favorables au petit gibier étaient générateurs de nombreux emplois, les propriétaires étaient parvenus à « intéresser », «enrôler » et « mobiliser» les paysans au sein de réseaux gravitant autour de l'organisation de la chasse sur ces domaines privés.

Mirloup (1975) et Chardon (1993) nous décrivent les pratiques cynégétiques prévalant en Sologne jusque dans les années 1970 comme majoritairement associées à la présence du petit gibier, et notamment aux pratiques intensives d'élevages de faisans. Cela les conduisait à conclure que la Sologne, jusqu'en 1970, dépendait exclusivement de la chasse. Bien que ne disposant pas de données chiffrées plus récentes, nous avons néanmoins recueilli lors du travail d'enquête de nombreux témoignages indiquant un sentiment généralisé de grande dépendance économique vis-à-vis de l'activité cynégétique.

\section{La dynamique de l'engrillagement en Sologne}

Il y a trente ans déjà, Gillardot (1981) notait l'apparition d'un nouveau type de clôtures, qui ne servait plus à tenir le bétail à l'écart des parcelles cultivées. Ces nouvelles clôtures forestières avaient pour but de marquer les limites des propriétés privées, d'interdire aux promeneurs l'accès aux propriétés et permettaient le cantonnement des populations de gibier à l'intérieur des domaines. Par la suite, Chardon (1993) a également fait état de ces grillages qu'elle considérait comme des extravagances, associées à des nouveaux accédants non solognots. L'étude de 2011, commanditée par le pays de Grande Sologne, la lecture de la presse locale, la réalisation d'un film (Gasselin, 2011) et la création d'une page Facebook fédérant des commentaires anti-clôtures permettaient de penser que la propagation des engrillagements forestiers en Sologne s'était récemment accélérée, et qu'il s'agissait d'une pratique suffisamment inédite pour susciter la controverse. Ainsi, le 22 mars 2012, un journaliste de la presse locale titrait : « La Sologne à $98 \%$ contre l'engrillagement » (Aucante, 2012), ce qui interroge sur l'identité et les motivations des $2 \%$ restants qui érigent les grillages. De l'avis de nombreux enquêtés, l'origine de ces pratiques serait à rechercher parmi les nouveaux accédants non solognots qui chercheraient ainsi à affirmer leur statut de propriétaire. Néanmoins, nous avons pu documenter plusieurs cas où la décision d'installer des grillages a été prise par des propriétaires ayant hérité de biens familiaux. Mais si l'engrillagement n'est pas une pratique allogène importée en Sologne par des nouveaux accédants étrangers désireux d'enclore hermétiquement leur bien, d'où vient-il et quelles en sont les causes? Alors que la société solognote au début du $\mathrm{X} \mathrm{X}^{\mathrm{e}}$ siècle nous est décrite comme fondamentalement organisée autour de la grande propriété privée à visée cynégétique (Chardon, 1993), un siècle plus tard, ce sont précisément des arguments liés à la privatisation du gibier et aux pratiques cynégétiques qui étaient présentés par les médias comme éléments structurants de l'argumentaire anti-grillages (Gasselin, 2011). Il convient ici de rappeler le statut légal du gibier : en tant que res nullius, tout animal encore vivant n'appartient à personne tant qu'il n'a pas été tué - sauf dans le cas des enclos de chasse, répondant à des critères très stricts, et minoritaires sur le territoire ( $3 \%$ de la surface, d'après l'Office national de la chasse et de la faune sauvage [ONCFS]). En dépit du droit des propriétaires privés à s'enclore, les opposants aux grillages interviewés par les médias évoquaient volontiers le fait que la grande faune sauvage représentait un bien commun à préserver. Dans la mesure où la controverse médiatique portait en grande partie sur la viabilité, à terme, des populations d'ongulés sauvages confrontés à ces grillages, il nous a semblé 
logique de commencer par étudier les effets des engrillagements sur les populations de grand gibier.

\section{Les engrillagements et leurs effets sur les populations de grand gibier}

Les grands ongulés évoluent au sein d'espaces vitaux relativement vastes : entre 1000 et 3000 ha pour le cerf (Patthey, 2003) et entre 400 et 1000 ha pour le sanglier (Saïd et al., 2012). C'est pourquoi cerfs et sangliers sont susceptibles d'établir leurs domaines vitaux à cheval sur plusieurs propriétés et, le cas échéant, de voir leurs déplacements entravés par les limites de propriétés grillagées. C'est le cas à plus forte raison si ces engrillagements sont hermétiquement clos, ce qui, on l'a vu (Fig.), est loin d'être systématique. L'analyse des médias et les résultats d'enquêtes indiquent que l'accusation de constituer " une entrave à la circulation du grand gibier » est un grief largement partagé contre ces installations. Dans le cadre de notre projet interdisciplinaire, nous avons donc tout d'abord cherché à caractériser la structuration génétique des populations de cerfs, à l'échelle de la Sologne, en vue d'appréhender les effets des grillages sur leurs déplacements. Ces travaux indiquent que l'obstacle majeur à la circulation des cerfs en Sologne est l'autoroute A71 qui traverse la région sur un axe nord/sud (expliquant $20 \%$ de la variabilité observée). Néanmoins, dans la partie ouest de la Sologne, les engrillagements semblent également contraindre les déplacements de ces animaux, puisque $1 \%$ de la variabilité génétique observée s'explique par la présence de clôtures (Masson, 2015). S'ajoutant au reproche strictement lié à l'effet d'obstacle, les engrillagements sont souvent considérés comme générateurs de surconcentrations locales d'animaux, tant par nos enquêtés que dans les médias. Nous avons donc consacré un autre volet de notre travail à l'étude des effets des grillages sur les concentrations locales d'animaux (Baltzinger et al., 2016). Nous avons constaté que les grillages non clos ne constituent pas un obstacle à la circulation du grand gibier, dans la mesure où les traces de passages d'animaux sont réparties uniformément et indépendamment de la géométrie de ces installations ; d'un autre côté, nous avons mis en évidence la fidélité des cerfs à leurs sites de repos, qui sont préférentiellement localisés à l'intérieur d'espaces partiellement clos. Dans la mesure où le cerf, en tant que ruminant, consacre au moins la moitié de son temps à cette activité, la localisation des sites de repos joue un rôle déterminant dans la répartition temporelle et spatiale des animaux, au sein des paysages. Ce constat est en conformité avec les témoignages de nos enquêtés : les dispositifs partiellement clos seraient donc conçus pour concentrer le gibier, la plupart du temps, aux endroits où l'on souhaite qu'il demeure. « La plupart du temps » semble ici jouer un rôle crucial, dans la mesure où les grillages partiellement clos - laissant les animaux entrer et sortir - semblent, aux yeux des personnes interviewées, beaucoup plus problématiques que ceux qui sont complètement imperméables, comme a pu nous l'expliquer ce membre de la fédération des chasseurs : « S'il y a clôture, il faut qu'elle soit hermétique ! C'est-àdire pas de piège à animaux [...] Il y en a qui laissent faire des petits trous ou qui les aménagent pour que les animaux rentrent mais ne ressortent pas » (Siegfried). De l'avis de nombreux enquêtés, les grillages partiellement clos sont attractifs pour les animaux, et ce d'autant plus qu'ils s'accompagnent le plus souvent d'un ensemble de pratiques telles que l'agrainage et la surveillance par un ou des gardes afin d'éviter l'intrusion humaine et ainsi assurer la « tranquillité » (le non-dérangement) aux animaux. C'est ainsi qu'il faut comprendre les propos de ce propriétaire ayant engrillagé la quasi-totalité de son périmètre : «Ce qui est très intéressant, $c^{\prime}$ est des animaux qui bougent et comme peut-être qu'ils sont tranquilles et qu'il y a de la nourriture, ils viennent et [...] $c^{\prime}$ est pour ça qu'on voit de plus en plus de cerfs, le gibier rentre et si il se trouve bien ici, il rentre et il reste " (Silviano). Le but est de « cantonner » les animaux, c'està-dire de leur "donner une habitude de territoire " (Sylvie). Ainsi nos résultats écologiques croisés avec les enquêtes socioanthropologiques indiquent que cerfs et sangliers se concentrent au sein d'espaces partiellement clos de grillages, plus par incitation que par contrainte. Loin d'être une simple nuance, la différence entre « parc de chasse », qui sédentarise les animaux par la contrainte, et grillage partiellement clos, qui les conduit à élire un domaine vital plutôt qu'un autre, semble fondamentale pour la plupart des enquêtés. Ce point s'exprime, par exemple, dans la différence manifeste que ce propriétaire, lui-même engrillagé sur la quasi-totalité de son périmètre, établit entre ses propres engrillagements et ceux des " parcs »: «Y'a plus de côté sauvage, y'a plus rien du tout, c'est fini! Non mais c'est complètement perdu ! C'est comme la chasse dans un parc, y'a plus de côté sauvage, c'est fini » (Samson). De l'avis de tous, la liberté de circuler est à la fois cause et conséquence du caractère "sauvage » des animaux ou du paysage : «Ça paraît être un espace de liberté parce que ça a l'apparence de la sauvagerie » (Serge). De là découlent de nombreuses considérations sur le caractère « éthique » des pratiques de chasse en territoire engrillagé.

\section{Les engrillagements comme mode de chasse}

Lors des enquêtes, les défenseurs de l'engrillagement ont presque toujours commencé par nous les présenter comme des outils servant à diriger les animaux le long d'itinéraires privilégiés, dans le but de limiter les accidents de la route notamment, et en particulier les jours de chasse (Poinsot et Saldaqui, 2009). Ce qui est vrai pour les animaux chassés l'est également pour les chiens de 
chasse qui les suivent. De façon moins altruiste, le fait d'être en mesure de diriger les flux d'animaux permet de les suivre plus aisément, ce qui facilite la pratique de la chasse à courre. Plus généralement, l'engrillagement facilite les pratiques de chasse, y compris en battue. En particulier, la construction de miradors aux points de passage obligés laissés ouverts dans le grillage, ou même la fermeture épisodique de ces ouvertures au moyen de rubalises (rubans de balisage) les jours de chasse, semblent être des pratiques courantes. Ce mode de chasse est décrit par Édith Chardon (1993) comme un héritage datant de la fin du XIX ${ }^{\mathrm{e}}$ siècle, lorsque le sanglier était indésirable en Sologne : " Les fermés consistent à aller poser la veille des hectomètres de banderoles (ficelle sur laquelle sont attachés des morceaux de tissu ou de plastique blancs, rouges ou jaunes) de préférence la nuit lorsque les animaux paissent autour d'un bois dans lequel a été repérée l'entrée de quelques animaux à l'aide des "revoirs" ${ }^{6 "}$. Au matin, les chasseurs sont postés à des places précises tandis que les rabatteurs et les chiens battent les bois afin de pousser les animaux vers les tireurs ».

En raison de ce qui précède, on comprend pourquoi l'engrillagement permet d'influencer la gestion des populations de gibier sur des surfaces excédant largement le territoire strictement possédé en propre par le chasseur : d'un côté, les animaux se concentrent la plupart du temps dans les espaces partiellement clos, d'où il est quasiment impossible pour un voisin chasseur de les déloger, mais de l'autre, les grillages facilitent au contraire les pratiques de chasse pour ceux qui sont en mesure de maîtriser les flux d'animaux (notamment en permettant une fermeture complète les jours de chasse). Ainsi, nous avons, par exemple, pu documenter un cas de conflit ouvert entre un chasseur dont la propriété est partiellement close de grillages et son voisin forestier qui subit la pression d'herbivorie des cerfs sur ses peuplements forestiers. Ce dernier était de fait dans l'incapacité de chasser lui-même les cerfs présents de façon intermittente sur sa propriété en raison des grillages non clos installés par son voisin. Nous avons également recueilli un grand nombre de propos à première vue paradoxaux qui attribuent l'augmentation générale des populations de gibier aux engrillagements, tout en accusant les propriétaires engrillagés de se livrer à des massacres à grande échelle. C'est ce sentiment ambivalent qui s'exprime dans les propos de ce gérant de propriété partiellement engrillagée, à propos des pratiques de son employeur : «Il y a beaucoup de sangliers maintenant, plus qu'avant [...] Il faut bien prélever les sangliers! Nous, on essaye de respecter, quoi ! [...] À un moment donné, il faut en

\footnotetext{
6 Les revoirs sont des zones de faible surface régulièrement retournées de façon à rester meubles, afin que toute trace de passages d'animaux puisse $\mathrm{s}^{\prime} \mathrm{y}$ imprimer.
}

faire aussi ! Sur le nombre, on peut pas en faire dix et puis on s'arrête, des fois, il peut y avoir des débordements... » (Sven). Envisagée du point de vue d'une habitante non propriétaire, une situation analogue est décrite dans ces termes : « Ça tirait à tout bout de champ, je croyais que c'était un ball-trap, mais finalement c'était pas du tout un ball-trap vu la pétarade, et [...] ils étaient en train de coincer les cochons contre les grillages et de les tirer comme des... » (Sylvie). Loin d'être anecdotiques ou accidentels, les dispositifs de « clôtures en $\mathrm{U}$ » ou en « entonnoirs » sont très répandus sur le territoire et tout à fait intentionnels (Fig.). Ils permettent à ceux qui les érigent d'imposer leurs objectifs de gestion cynégétique à leur voisinage dans la mesure où l'engrillagement et les diverses pratiques associées rendent leur propriété plus attractive que celle de leurs voisins et leur assurent une maîtrise totale des flux d'animaux les jours de chasse.

\section{Les évolutions des populations de gibier et la fermeture du paysage}

Les enquêtés ont souvent manifesté leurs craintes quant à la viabilité des populations de grand gibier, reprenant un argument très médiatisé. Il convient ici de rappeler que, même si les cerfs et les sangliers sont présents en Sologne depuis plusieurs siècles - notamment sur les domaines de Chambord et de Cheverny -, leurs effectifs étaient très faibles il y a encore trente ans. Ce fait a été mentionné par la plupart de nos enquêtés. Les réalisations de plans de chasse indiquent une augmentation de $40 \%$ pour le cerf et de $65 \%$ pour le sanglier sur l'ensemble de la Sologne entre 1973 et 2012, ce qui représente environ le double de l'augmentation nationale sur la même période ( $24 \%$ pour le cerf, $39,9 \%$ pour le sanglier ${ }^{7}$ ). Alors que Mirloup en 1975 et Chardon en 1993 nous décrivaient une économie cynégétique régionale basée sur le petit gibier, en 2014, les tableaux de chasse sont composés quasi exclusivement de cerfs, de sangliers et de chevreuils qui pourtant continuent à voir leurs effectifs croître. Durant la même période, la chasse au petit gibier semble avoir complètement disparu. De l'avis de nombreux enquêtés, la présence d'ongulés sauvages et notamment de sangliers est considérée comme incompatible avec les populations de faisans ou de perdrix nichant au sol ou dans les strates basses, en raison de la prédation exercée sur les nids. Néanmoins, rien ne permet de penser qu'il s'agisse plus d'une incompatibilité « écologique » que d'une incompatibilité entre les modes de gestions associés au gibier à poil ou à plumes (Baltzinger et al., 2016). En effet, c'est sur les pratiques d'élevage intensif du faisan que la chasse reposait en Sologne jusque dans les années 1970 (Mirloup, 1975).

\footnotetext{
7 Source : réseau Ongulés sauvages 2012.
} 
L'abandon de ces pratiques, couplé à l'effet d'aubaine lié à l'augmentation rapide des populations d'ongulés sauvages, explique très probablement en grande partie le remplacement du gibier à plume par le gibier à poil. Cette concomitance entre augmentation des populations d'ongulés et abandon des pratiques d'élevage pourvoyeuses d'emplois laisse, de plus, supposer que ce phénomène a eu des répercussions sociales importantes en Sologne.

\section{Des mobilités inégales : les engrillagements comme filtres sociaux}

Une fois les domaines engrillagés, il est possible d'avoir un contrôle quasi absolu sur les flux humains, de choisir qui est présent sur la propriété et quand. En régulant ainsi les flux humains, le propriétaire est en droit $\mathrm{d}^{\prime}$ attendre que les personnes qu'il n'a pas explicitement invitées s'abstiennent d'entrer ou, pour le moins, qu'elles lui adressent une demande qu'il aura toujours la possibilité de refuser : "Si je vous vois de temps en temps et que vous êtes gentille et bien élevée [...] Si les gens sont corrects et bien élevés, c'est possible » (Samson). En outre, cette même personne peut poursuivre quelques minutes plus tard, au sujet des « écologistes »: « C'est des gens qui prétendent connaître la nature parce qu'ils prétendent l'avoir appris dans les livres. Or la nature ça s'apprend dans deux endroits : dans les livres et sur le terrain et si vous avez pas une expérience terrain, tout ce que vous avez appris dans les livres c'est pas suffisant »(Samson). Nous avons plusieurs fois rencontré, de la part des propriétaires engrillagés, cet argumentaire s'appuyant sur (i) la revendication du droit de choisir qui est présent et quand sur « le terrain », articulée avec (ii) des considérations au sujet de la pratique du «terrain » qui serait indispensable à la connaissance fine du lieu et aboutissant à la conclusion que (iii) seuls les propriétaires peuvent revendiquer un statut de connaisseur et de protecteur de la nature sur leur propriété. Cet argumentaire fonctionne d'autant mieux à l'encontre des citoyens ordinaires, censément non initiés à la nature, ce qui s'entend dans les propos de ce propriétaire visant à expliquer sa décision d'engrillager : " La personne vraiment à qui ça appartient, il sait qu'il faut qu'il fasse attention, il a une gestion à faire. Et la personne qu'a pas appris au moins cette éducation-là, en disant au moins faut que j'en prenne soin, ben il sait pas, quoi ! Mais même si euh, on essaie, hein ! On essaie de dire aux gens euh, faites pas ça, faites attention... » (Saturnin). Cette connaissance du milieu ainsi que le souci permanent de «bien gérer » quelque chose qui leur appartient en propre seraient donc le garant de pratiques adaptées de la part des propriétaires.
De plus, l'engrillagement permet d'exclure les « pollueurs ", notamment ceux qui "dérangent le gibier ». Dans la mesure où l'engrillagement est associé au contrôle des flux humains, c'est tout naturellement que la volonté des propriétaires de modifier les cheminements et les itinéraires s'immisce dans les discours. Un grand nombre de domaines solognots sont, en effet, traversés par des chemins communaux. La question de l'accès aux chemins communaux a très souvent été évoquée lors des entretiens, en lien avec les engrillagements : «Ce qui se vend le plus cher, c'est une propriété close, sans chemin de commune. Eh oui, c'est évident!» déclare, par exemple, Sylvestre. Sur un gradient décroissant de légalité, des panneaux au contenu diversement accueillant sont disposés à l'entrée des domaines, puis à intervalles réguliers le long des chemins de commune ; des altercations physiques parfois musclées nous ont également été rapportées, de même que la pose tout à fait illégale de chaînes barrant l'accès à ces chemins. Décourageant les promeneurs, ces pratiques s'expliquent en partie par le souhait de garantir une tranquillité maximale au grand gibier au sein des espaces partiellement clos. Sur le même registre de l'intimidation, la menace de l'engrillagement peut être utilisée dans le but de discipliner un voisin récalcitrant, ou de façon punitive suite à un différend. Dans ce cas, la teneur de la menace relève de « l'effet aspirateur » qui permet, grâce au grillage, de confisquer une grande partie du gibier au voisin, par exemple : «Vous êtes mon voisin, vous me plaisez pas, je vous mets le grillage! Si vous avez un grillage, vous pouvez plus rien dire parce que $[. ..] y^{\prime}$ a certaines choses que vous pourrez plus faire et qui perdront de la valeur, en l'occurrence y'a la chasse... » (Sylas). L'engrillagement manifeste aussi ostensiblement la surface que $l^{\prime}$ 'on possède ; il est de plus cher à poser et à entretenir : «Non, mais ce qu'on sait quand on voit des clôtures, de toute façon, c'est que c'est des gros propriétaires [...] Ils ont plus de mille hectares et ils veulent bien marquer leur territoire. C'est une rivalité entre eux ! Moi j'ai ma propriété en Sologne, elle fait tant d'hectares, ben tiens, moi j'achète celle d'à côté, elle fait tant d'hectares!» (Sacha). Cette richesse réelle ou fantasmée manifestée par l'engrillagement laisse un goût d'autant plus amer dans la bouche des résidents qu'ils expriment par ailleurs fortement leur sentiment de dépendance économique vis-à-vis des riches propriétaires : «Après, ouais, comme je te dis, $y^{\prime}$ a tellement d'enjeux économiques derrière aussi, que voilà, quoi ! [...] Ca fait vivre des villages, quoi, enfin ces gens-là même s'ils se clôturent... même si c'est des gros cons et tout ça, bah, quand même ils font vivre, ils font vivre des gens, quoi, donc... Mais bon, après ils pourraient très bien faire vivre les gens sans se clôturer, quoi ! » (Sébastien). 


\section{L'engrillagement révélateur des compétences sociales du gibier}

Comme on 1'a vu plus haut, le débat autour de l'engrillagement semblait porter sur les effets de ces installations sur le grand gibier. Nous avons montré que cet effet est bien réel même si la concentration des animaux au sein des espaces partiellement clos est plurifactorielle et s'explique le plus souvent par un ensemble de pratiques associées. Il n'en demeure pas moins vrai que le grillage est un outil décisif dans l'organisation des journées de chasse et qu'il permet de se fixer et d'atteindre des objectifs cynégétiques sans dépendre de ses voisins, voire d'imposer ses propres objectifs à tout le voisinage.

Dans ce système, tous considèrent la liberté de circulation du gibier comme primordiale. Seul un animal libre de choisir son territoire peut être considéré comme " sauvage »: " sauvage, c'est sauvage, oui, c'est ça, laisser la nature circuler librement » (Sarah). Aussi étonnant que cela puisse paraître, les pratiques d'agrainage au maïs - parfois en quantités considérables - ne sont jamais envisagées comme incompatibles avec le statut « sauvage » du gibier. Cela transparaît dans les propos de ce propriétaire qui reconnaît lui-même par ailleurs pratiquer l'agrainage au maïs à destination du sanglier : "La chasse s'est un peu transformée... Les chasses au faisan qui sont devenues des chasses d'élevage ! Ça perdait un peu de son intérêt... Moi, j'ai été passionné par la chasse mais la chasse naturelle, la chasse sauvage avec un chien : on a arrêté la chasse au petit gibier » (Sigisbert). En Sologne comme ailleurs, un animal « sauvage » est un animal « fuyard », qui à ce titre doit posséder un réflexe de fuite et être physiquement en capacité de le faire de façon efficace (Clutton-Brock, 2002). Contrairement à l'élevage très artificiel du petit gibier souvent considéré comme incapable de produire des animaux véritablement fuyards, l'agrainage du gros gibier est effectué avec une rythmicité variable, parfois à l'aide d'agrainoirs automatiques, ce qui limite encore les occasions de contact entre les humains nourriciers et le grand gibier « sauvage ». Ainsi, les grillages partiellement clos ne remettent pas fondamentalement en cause le caractère fuyard et sauvage du gibier, mais permettent cependant de maintenir les pratiques cynégétiques privatives caractéristiques de la Sologne. Cette chasse privée au grand gibier sauvage et fuyard ne va pas de soi dans la mesure où il s'agit d'espèces évoluant en principe sur des surfaces débordant largement les limites des propriétés privées. Ces dispositifs permettent en fait de pratiquer la chasse au gros gibier en petites équipes triées sur le volet, tout en garantissant au gibier sa liberté de circulation et donc son caractère sauvage.

Allant jusqu'à permettre la confiscation du pouvoir de chasser, les engrillagements nous révèlent ainsi à quel point le gibier, en élisant un territoire, conforte les grands propriétaires terriens engrillagés dans leur statut d'élite sociale, comme l'exprime cet agent immobilier : «Ce qui donne la valeur, ça va être l'aménagement qui a été fait sur cette propriété, le gibier, le nombre de bracelets qui y a été délivré » (Samuel). De plus, le caractère sauvage du gibier déteint sur son territoire d'élection qui devient sauvage à son tour (Bobbé, 2000). Dans la mesure où la possibilité d'établir des contacts réguliers avec le sauvage à travers la pratique de la chasse est considérée, par nos enquêtés de Sologne et plus généralement en Occident, comme un privilège social (Hell, 1994), l'engrillagement, en incitant les animaux sauvages à élire un territoire sans trop les contraindre, peut être compris comme un dispositif $\mathrm{d}^{\prime}$ " ascenseur social». Ainsi, le propriétaire d'un « bon territoire de chasse » est en mesure d'intégrer, via le système des invitations données puis rendues, le réseau fermé des propriétaires solognots. «Comme j'avais des domaines... j'avais des grosses chasses, que je louais... ça me faisait connaître $\mathrm{d}^{\prime}$ autres personnes, ou même les voisins des voisins [...] Ils vous disent : j'ai une propriété là-bas. Moi, je les invite chez moi, eux, ils me réinvitent chez eux. C'est des échanges » (Savin). Sur ce point, les témoignages de nos enquêtés quant à l'indexation des prix de l'immobilier sur la valeur cynégétique des territoires sont, eux aussi, tout à fait significatifs.

\section{Conclusion : les engrillagements et le pouvoir}

Nous avons vu que les engrillagements, ainsi que les autres pratiques humaines qui leur sont associées, ont pour effet de modifier les déplacements des animaux et la façon dont ils utilisent l'espace. Nous avons plus largement décrit la façon dont ces installations redéfinissent les mobilités et les modalités de mise en relations entre les êtres, qu'ils soient humains ou non humains. À travers ces redéfinitions, les engrillagements permettent à ceux qui les contrôlent de s'instituer en points de passage obligés au sein de nouveaux réseaux d'acteurs, et ce faisant de rompre les anciennes alliances (Callon, 1986). En effet, le réseau d'alliances en Sologne, jusque dans les années 1970, s'organisait autour de la grande propriété privée à vocation cynégétique, dédiée à la chasse au petit gibier ; cette activité cynégétique d'alors était pourvoyeuse de nombreux emplois pour l'élevage des animaux, la mise en culture des terres et l'organisation proprement dite des journées de chasse. Cela avait permis aux propriétaires $\mathrm{d}^{\prime}$ " intéresser », « enrôler » et « mobiliser » les acteurs humains et non humains de Sologne au sein de réseaux gravitant autour de ces grands domaines privés à vocation cynégétique. Pourtant, les populations de petit gibier ont récemment laissé place à la grande faune; les pratiques de gestion cynégétiques qui en 
découlent sont beaucoup moins coûteuses en personnel, notamment parce qu'il s'agit d'animaux qui s'alimentent généralement de façon plus autonome que le petit gibier. En cela, certaines alliances anciennes sont rompues, comme celle qui existait entre les propriétaires et leurs employés agricoles, ou celle qui associait les humains au petit gibier. Dans le même temps, se noue une alliance entre humains et grand gibier, qui fait intervenir deux nouveautés : il s'agit d'animaux sauvages et à grand domaine vital. Or, comme cela a déjà été démontré sur d'autres terrains (Mounet, 2012 ; Poinsot et Saldaqui, 2009), changer d'espèce de gibier nécessite des reconfigurations sociospatiales. En Sologne, le caractère sauvage du grand gibier permet aux grands propriétaires de s'exonérer d'une série de pratiques d'élevage coûteuses jugées artificielles et notamment d'un grand nombre d'emplois locaux. Pourtant, la plupart des domaines, aussi vastes soient-ils, ne suffisent pas à eux seuls à contenir les domaines vitaux d'animaux tels que cerfs, chevreuils et sangliers, qui se déplacent donc entre propriétés. En cela, le caractère sauvage pourrait sembler en contradiction avec le caractère privé de la propriété solognote à vocation cynégétique. En fait, c'est parce que le gibier chassé est sauvage, doit rester sauvage, qu'il est possible de présenter les grillages privés, non pas comme des obstacles étanches pour le gibier, mais au contraire comme des écrans protecteurs, assertions corroborées par les observations de gibier se concentrant la plupart du temps au sein d'espaces partiellement clos. Les engrillagements peuvent ainsi apparaître comme des dispositifs, utilisés par les propriétaires désirant chasser le grand gibier de façon privative, qui leur permettent, tout en gravitant autour de l'idée consensuelle de propriété privée à caractère cynégétique, de déployer ces deux nouveautés à leur avantage.

\section{Références}

Amelot X., André-Lamat V., Couderchet L., 2014. L'espace protégé ou la nature enfermée, in Laslaz L., Gauchon C., Duval M., Héritier S. (Eds), Les espaces protégés. Entre conflits et acceptation, Paris, Belin, 127-142.

Aucante P., 2012. La Sologne à $98 \%$ contre l'engrillagement, Le Petit Solognot, 22 mars 2012.

Ballif F., Rosière S., 2009. Le défi des «teichopolitiques». Analyser la fermeture contemporaine des territoires, L'Espace géographique, 38, 3, 193-206.

Baltzinger M., Mårell A., Archaux F., Pérot T., Leterme F., Deconchat M., 2016. Overabundant ungulates in French Sologne? Increasing red deer and wild boar pressure may not threaten woodland birds in mature forest stands, Basic and Applied Ecology, doi:10.1016/j.baae.2016.04.005.

Benjaminsen T.A., Svarstad H., 2009. Qu'est-ce que la « Political ecology » ?, Natures Sciences Sociétés, 17, 1, 3-11.
Bergerud A.T., Jakimchuk R.D., Carruthers D.R., 1984. The buffalo of the North. Caribou (Rangifer tarandus) and human developments, ARCTIC, 37, 1, 7-22.

Bobbé S., 2000. Les nouvelles cultures du sauvage ou la quête de l'objet manquant. État de la question, Ruralia, 7, 159-173.

Brechin S.R., Fortwangler C.L., Wilshusen P.R., West P.C. (Eds), 2003. Contested nature. Promoting international biodiversity with social justice in the twenty-first century, Albany, State University of New York Press.

Callon M., 1986. Éléments pour une sociologie de la traduction. La domestication des coquilles Saint-Jacques et des marinspêcheurs dans la baie de Saint-Brieuc, L'Année sociologique, 36, 169-208.

Callon M., Latour B., 2006. Le grand Léviathan s'apprivoise-til ?, in Akrich M., Callon M., Latour B. (Eds), Sociologie de la traduction. Textes Fondateurs, Paris, Presses des mines, 1132. Traduit de: Unscrewing the big Leviathan; or how actors macrostructure reality, and how sociologists help them to do so?, in Knorr-Cetina K., Cicourel A.V. (Eds), 1981. Advances in social theory and methodology. Toward an integration of micro- and macro-sociologies, London, Routledge \& Kegan Paul, 277-303.

Chardon É., 1993. Les conflits d'usages sur un territoire. L'exemple de Saint-Viâtre (Sologne). Thèse de doctorat en sociologie, Nanterre, Université Paris 10.

Clutton-Brock J., 2002 [1 ${ }^{\text {re }}$ éd. 1994]. The unnatural world. Behavioural aspects of humans and animals in the process of domestication, in Manning A., Serpell J.A. (Eds), Animals and human society. Changing perspectives, London/New York, Routledge, 23-35.

Fahrig L., 2003. Effects of habitat fragmentation on biodiversity, Annual Review of Ecology Evolution and Systematics, 34, 487-515.

Froissart Y., 2011. Faire face aux engrillagements en milieu naturel, agricole et forestier en Grande Sologne. Rapport d'étude, Trans-Formation Consultants, Lamotte Beuvron, Syndicat mixte du pays de Grande Sologne.

Gasselin X., 2011. Une maille de trop. Film documentaire, Paris, C'est à voir/Seasons.

Gillardot P., 1981. La Grande Sologne. Thèse de doctorat de géographie, Paris, Université Paris-1-Panthéon Sorbonne.

Goldman M.J., Nadasdy P, Turner M.D. (Eds), 2011. Knowing nature. Conversations at the intersection of political ecology and science studies, Chicago/London, University of Chicago Press.

Hell B., 1994. Le sang noir. Chasse et mythe du sauvage en Europe, Paris, Flammarion.

Holdo R.M., Fryxell J.M., Sinclair A.R.E., Dobson A., Holt, R.D., 2011. Predicted impact of barriers to migration on the Serengeti wildebeest population, PLOS ONE, 6, 1, http:/ / dx.doi.org/10.1371/journal.pone.0016370.

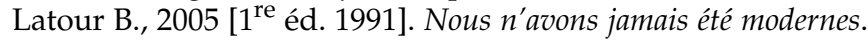
Essai d'anthropologie symétrique, Paris, La Découverte.

Masson M., 2015. Influence of habitat fragmentation and historical context on the genetic structure of red deer (Cervus elaphus). Mémoire de Master 2, Toulouse, Université Toulouse-3Paul Sabatier.

Mirloup J., 1975. La chasse en Sologne. Mémoire de maîtrise, Orléans, Centre régional de documentation pédagogique. 
Mouche J., 2013. Comment l'engrillagement en Sologne questionne$t$-il les valeurs associées à la forêt? Recherche exploratoire sur l'interaction homme-nature dans le contexte de la propriété privée. Mémoire de Master 2, Paris, AgroParisTech/ Muséum national d'Histoire naturelle.

Mounet C., 2012. Conflits et reconfigurations socio-spatiales autour du sanglier. Des postures générales aux arrangements locaux, Économie rurale, 327-328, 79-95.

Nielsen C.K., Nelson S.J., Porter W.F., 1997. Emigration of deer from a partial enclosure, Wildife Society Bulletin, 25, 2, 282-290.

Packer C., Loveridge A., Canney S., Caro T., Garnett S.T., Pfeifer M., Zander K.K., Swanson A., MacNulty D., Balme G., Bauer H., Begg C.M., Begg K.S., Bhalla S., Bissett C., Bodasing T., Brink H., Burger A., Burton A.C., Clegg B., Dell S., Delsink A., Dickerson T., Dloniak S.M., Druce D., Frank L., Funston P., Gichohi N., Groom R., Hanekom C., Heath B., Hunter L., Delongh H.H., Joubert C.J., Kasiki S.M., Kissui B., Knocker W., Leathem B., Lindsey P.A., Maclennan S.D., McNutt J.W., Miller S.M., Naylor S., Nel P., Ng'weno C., Nicholls K., Ogutu J.O., Okot-Omoya E., Patterson B.D., Plumptre A., Salerno J., Skinner K., Slotow R., Sogbohossou E.A., Stratford K.J., Winterbach C., Winterbach H., Polasky S., 2013. Conserving large carnivores: dollars and fence, Ecology Letters, 16, 5, 635-641.

Patthey P., 2003. Habitat and corridor selection of an expanding red deer (Cervus elaphus) population. Thèse de doctorat,
Lausanne, Faculté des sciences de l'Université de Lausanne.

Poinsot Y., Saldaqui F., 2009. Quelle organisation territoriale pour une gestion durable des sangliers ? Un exemple pyrénéen, M@ppemonde, 94, 2, http://mappemonde.mgm.fr/ num22/articles/art09203.html.

Razac O., 2009. Histoire politique du barbelé, Paris, Flammarion.

Rodary E., Bologna V., 2014. Politiser la connectivité, in Laslaz L., Gauchon C., Duval M., Héritier S. (Eds), Les espaces protégés. Entre conflits et acceptation, Paris, Belin, 143-157.

Saïd S., Tolon V., Brandt S., Baubet E., 2012. Sex effect on habitat selection in response to hunting disturbance: the study of wild boar, European Journal of Wildlife Research, 58, 1, 107-115.

Somers M.J., Hayward M. (Eds), 2012. Fencing for conservation. Restriction of evolutionary potential or a riposte to threatening processes?, New York, Springer.

Strayer D.L., Power M.E., Fagan W.F., Pickett S.T.A., Belnap J., 2003. A classification of ecological boundaries, BioScience, $53,8,723-729$.

Wallis De Vries M.F., 1995. Large herbivores and the design of large-scale nature reserves in Western Europe, Conservation Biology, 9, 25-33.

Webb S.L., Gee K.L., Wang G., 2010. Survival and fidelity of an enclosed white-tailed deer population using capturerecapture-reporting data, Population Ecology, 52, 1, 81-88.

Reçu le 30 juin 2015. Accepté le 20 mai 2016. 